\title{
BACTERIOLOGICAL STUDY OF PATIENTS WITH CSOM TREATED EMPIRICALLY USING ANTIBIOTICS AND REPORTING TO A TEACHING HOSPITAL OF NORTHERN KERALA
}

\author{
S. Muneeruddin Ahmed ${ }^{1}$, A. Siva Kumar ${ }^{2}$ \\ 1 Professor, Department of ENT, Kannur Medical College, Anjarakandy, Kannur, Kerala. \\ 2Professor, Department of ENT, Kannur Medical College, Anjarakandy, Kannur, Kerala.
}

ABSTRACT
BACKGROUND
Chronic Suppurative Otitis Media (CSOM) is caused by a mixed bacterial infection. In India, the trend is to start an empirical
treatment in patients presenting with history of discharge from the ear before a lab report on culture of pus is available. This study
is to note the effect of general practice of choosing the empirical antibiotics, both systemic and topical to treat CSOM patients by
isolating and culture of organism in CSOM; to investigate their sensitivity to various antibiotics.

\section{MATERIALS AND METHODS}

212 patients attending the ENT Department of a Teaching Hospital in Northern Kerala with history of discharge from the ears and already using antibiotics were included for this study. Culture swabs were taken from all patients suffering from CSOM (both types) and sent to the Microbiology Department for processing. The specimens were cultured and the isolates were identified using standard microbiological techniques. The antibiotic susceptibilities of the isolates were determined with standard antibiotic discs using the disc diffusion method.

\section{RESULTS}

This study analyses the trends of using antibiotics by the practitioners in the community, isolation and culture of causal organisms and their sensitivity to various antibiotics. The major organisms isolated were Methicillin sensitive Staphylococcus aureus [MSSA] (48.21\%) followed by S. aureus (MRSA) 18.75\%, Pseudomonas aeruginosa (12.50\%) and Klebsiella (7.14\%). The sensitivity of S. aureus (MSSA) was $52 \%$ to Amoxicillin, 58.2\% to Amoxicillin and clavulanic acid combination. Staph MRSA showed $100 \%$ sensitivity to the same antibiotics respectively. Ceftazidime and Cephalothin were effective against Pseudomonas in 73.4 and $66.8 \%$ respectively. Topical antibiotics like Gentamicin showed susceptibility rate against all the organism of CSOM ranging from 22 to $86.2 \%$, followed by Chloramphenicol ranging from 18.6 to $71 \%$.

\section{CONCLUSION}

The study of microbial pattern and their antibiotic sensitivity determined the prevalent bacterial organisms causing CSOM in the community where empirical antibiotics are used. The information is useful in treating CSOM and its complications for a successful outcome and thus to prevent the emergence of resistant strains.

\section{KEYWORDS}

CSOM, Culture, Antibiogram, Antibiotic, Bacteria, Staphylococcus.

HOW TO CITE THIS ARTICLE: Ahmed SM, Kumar AS. Bacteriological study of patients with CSOM treated empirically using antibiotics and reporting to a teaching hospital of Northern Kerala. J. Evolution Med. Dent. Sci. 2017;6(22):1763-1766, DOI: $10.14260 /$ Jemds/2017/388

\section{BACKGROUND}

Chronic Suppurative Otitis Media (CSOM) is defined as a chronic inflammation of the Mucoperiosteum of the middle ear cleft presenting with recurrent discharge from the ears in the presence of tympanic membrane perforation. The natural history of the disease, usually beginning in childhood 1,2 as a tympanic membrane perforation due to Acute Suppurative Otitis Media (ASOM) or less commonly due to Otitis Media. . $^{3-5}$

In India, the CSOM was observed commonly during the first 6 years of a child's life with a peak around 4 years. ${ }^{6}$ Patients with tympanic perforations which continue to

Financial or Other, Competing Interest: None.

Submission 06-02-2017, Peer Review 01-03-2017,

Acceptance 08-03-2017, Published 16-03-2017.

Corresponding Author:

S. Muneeruddin Ahmed,

Professor,

Department of ENT,

Kannur Medical College,

Anjarakandy, Kannur, Kerala.

E-mail: ahmedmunirnet@gmail.com

DOI: $10.14260 /$ jemds $/ 2017 / 388$ discharge mucoid, purulent material for periods from 6 weeks ${ }^{7}$ to 3 months in spite of medical treatment are defined as CSOM. CSOM is a commonly encountered infection of the middle ear all over the world. In 1990, about 28,000 deaths all over the world and largely among developing countries were due to otitis media. 8,9 Mortality and disabilities due to otitis media are primarily related to the complications of CSOM, ${ }^{10}$ particularly brain abscess. ${ }^{11}$

The trends of using empirical antibiotics were observed in India by all practitioners who come across CSOM patients. Inadequate usage of antibiotics and untreated cases of CSOM result in major incidences of complications. Timely isolation of bacteria causing CSOM and the knowledge of the local pattern of infection in any given geographical area is essential to enable efficacious treatment of this disorder. The goal of the management is to achieve a safe, dry ear, eradicate disease and improve hearing. The present study hence attempts to analyse the trends in empirical usage of antibiotics and to determine the microbial profile (aerobic and anaerobic) and the antibiograms of active CSOM patients of Northern Kerala. 


\section{MATERIALS AND METHODS}

212 patients were enrolled in this study on outpatient nominal registration of ENT Department of a teaching Hospital of Northern Kerala. This retrospective study was conducted between December 2015 and January 2017.

\section{Inclusion Criteria}

1. Patients of all age groups with CSOM (both types) with tympanic membrane perforation and having purulent or mucopurulent discharge were included.

2. Patients who had used antibiotics empirically elsewhere were included.

3. Postoperative patients (Grommet insertion, Mastoidectomy) with surgery done elsewhere and continuing with discharge were included.

4. Patients using local antibiotic drops were included.

\section{Exclusion Criteria}

1. Patients with external ear conditions like external otitis, otomycosis and eczema were not included; 2 . Patients with immunocompromised diseases like diabetes, viral positive patients were not included. Clinical history of ear discharge, age, sex, duration of antibiotic treatment including both local and systemic usage was taken. Single use culture swabs were used to harvest the middle ear bacterial flora through the external auditory canal under microscope. Care was taken to avoid surface contamination and the swabs were transported to the Microbiology Department of the hospital for culture and sensitivity testing. All the 212 swabs were processed for aerobic and anaerobic organism using standard microbiological procedures. All organisms isolated were identified according to standard microbiological methods. Antibiotic susceptibility testing was carried out using the disc diffusion technique on agar and commercial antibiotic discs. The antibiotic discs used were Amoxicillin, Amoxicillin with Clavulanic acid, Amikacin, Cephalothin, Ceftazidime among the systemically used antibiotics and Chloramphenicol, Polymyxin, Ciprofloxacin, Gentamicin, Neomycin and Bacitracin among the topically used antibiotics. The antibiotic disc impregnated culture plates were incubated at 37 degrees overnight. The diameter of the zone of inhibition was measured and recorded as resistant or susceptible according to the National Committee for Clinical Laboratory Standards (NCCLS) interpretative criteria. ${ }^{12}$

\section{RESULTS}

\section{Observations}

Among the 212 patients, 147 were males and 65 were females. The male-to-female ratio was 1: 2.26. Patient's ages ranged between 3 years to 76 years with a mean age of 37.24 \pm 4.2 (Table 1$)$.

\begin{tabular}{|c|c|c|c|c|c|c|}
\hline $\begin{array}{l}\text { Age Group } \\
\text { in Yrs. }\end{array}$ & $\begin{array}{c}\mathrm{M} \\
147 \\
\end{array}$ & $\begin{array}{c}F \\
65 \\
\end{array}$ & $\begin{array}{c}\text { Culture Positive } \\
112 \\
\end{array}$ & $\begin{array}{c}\text { Culture } \\
\text { Negative } 99 \\
\end{array}$ & $\begin{array}{c}\text { Single } \\
\text { Organism- } 38 \\
\end{array}$ & $\begin{array}{c}\text { Multiple } \\
\text { Organism- } 74 \\
\end{array}$ \\
\hline $3-18$ & 20 & 12 & 10 & 22 & 4 & 6 \\
\hline $19-24$ & 34 & 13 & 23 & 24 & 8 & 11 \\
\hline $25-40$ & 39 & 18 & 34 & 23 & 7 & 27 \\
\hline $40-55$ & 24 & 07 & 21 & 10 & 6 & 15 \\
\hline $56-70$ & 16 & 09 & 13 & 12 & 8 & 7 \\
\hline 70 & 13 & 06 & 11 & 08 & 5 & 8 \\
\hline
\end{tabular}

The number of culture swabs positive for bacterial isolation and culture were $112(52.83 \%)$ and negative for $47.17 \%$. Among the positive cultures single organism was found in $33.93 \%$ and multiple organisms in $66.07 \%$ of the culture swabs (Table 1).

Among the positive isolations of culture swabs, Methicillin sensitive Staphylococcus aureus [MSSA] was isolated in 54 (48.21\%), Staphylococcus MRSA in 21 (18.75\%) followed by Pseudomonas aeruginosa (12.5\%), Klebsiella in 08 (7.14\%), Proteus mirabilis in 07 (6.25\%) and Escherichia coli in 07 (6.25\%) (Table 2).

\begin{tabular}{|c|c|c|}
\hline Organism & Number & \% \\
\hline Staphylococcus MSSA & 54 & $48.21 \%$ \\
\hline Staphylococcus MRSA & 21 & $18.75 \%$ \\
\hline Pseudomonas & 14 & 12.5 \\
\hline Klebsiella & 08 & 7.14 \\
\hline Proteus mirabilis & 07 & 6.25 \\
\hline E. coli & 08 & 7.14 \\
\hline
\end{tabular}

Table 2. Showing the Incidence of Different Organisms Isolated among the Positive Culture Swabs $(n=112)$
The antibiograms of the organisms isolated were tested and the results for the eleven most common organisms were studied. Among the antibiotics, 5 were systemically used antibiotics and 6 were topical antibiotics. Among the systemically used antibiotics Amoxicillin alone and in combination with Clavulanic acid were effective against Staph. MRSA organism with sensitivity ranging between 52 and $100 \%$. Ceftazidime and Cephalothin were effective against Pseudomonas in 73.4 and $66.8 \%$ respectively. All the topical antibiotics used in this study were effective against Staph. MSSA and MRSA organism with sensitivity ranging from 68 to $86.2 \%$. Their sensitivity against Pseudomonas was ranging between 18.6 to $68 \%$. Gentamicin was found to have the highest susceptibility rate against all the organism of CSOM ranging from 22 to $86.2 \%$ followed by Chloramphenicol ranging from 18.6 to $71 \%$. Polymyxin was effective by 20 to $68.6 \%$ and its sensitivity against pseudomonas was found to be $58.6 \%$, which was the highest among all other topical antibiotics. Ciprofloxacin was sensitive for CSOM organism ranging from 22 to $72.4 \%$ (Table 3). 


\begin{tabular}{|c|c|c|c|c|c|c|}
\hline \multirow[b]{2}{*}{ Antibiotics } & \multicolumn{4}{|c|}{ Organism } & \multirow[b]{2}{*}{ Proteus. m } & \multirow[b]{2}{*}{ E. coli } \\
\hline & Staph MSSA & Staph MRSA & Ps. aero genes & Klebsiella & & \\
\hline Amoxicillin & 52.0 & 100.0 & NT & 64.2 & 50.0 & 34.2 \\
\hline Amoxicillin+ Clavulanic & 58.2 & 100.0 & NT & 74.0 & 48.0 & 32 \\
\hline Amikacin & 36.4 & 100.0 & 41.0 & 55.0 & 49.0 & 30 \\
\hline Cephalothin & 41.4 & ND & 66.8 & 51.2 & ND & ND \\
\hline Ceftazidime & 44.8 & ND & 73.4 & ND & ND & ND \\
\hline Gentamicin & 86.2 & 22.0 & 28.0 & 35.0 & 66.0 & 27.2 \\
\hline Chloram-phenicol & 71.0 & 24.2 & 18.6 & 26.0 & 32.0 & 20.0 \\
\hline Polymyxin & 68.0 & 34.6 & 68.6 & 20.0 & 44.2 & 28.6 \\
\hline Ciprofloxacin & 72.4 & 40.0 & 22.0 & 24.0 & 49.0 & 32.0 \\
\hline Bacitracin & 83.4 & 38.0 & 19.8 & 17.4 & 21.6 & 18.4 \\
\hline Neomycin & 79.8 & 33.0 & 18.6 & 16.4 & 25.4 & 16.8 \\
\hline \multicolumn{7}{|c|}{ Table 3. Showing the Sensitivity Pattern of Common Organism of CSOM $(n=212)$} \\
\hline
\end{tabular}

The commonly used empirical antibiotics in the community by the practitioners for patients of CSOM in these parts of Kerala where Erythromycin, Ampicillin, Ciprofloxacin, Cephalexin, Clarithromycin and Amoxicillin and Cloxacillin combination. The duration of prescription ranged from 3 to 5 days.

\section{DISCUSSION}

CSOM is known for its Intracranial complications and one of the many factors contributing to their severity is the organism involved. Development of resistance to the antibiotics used against CSOM makes it further more difficult to treat their complications. ${ }^{13}$ Pathogenesis of CSOM complications is related to the bacterial antigens contributing to the osteolysis of bone resulting in local damage of middle ear. ${ }^{14}$ The complications range from brain abscess, lateral sinus thrombosis, meningitis and cerebellar abscess. mastoiditis, labyrinthitis, facial nerve paralysis are extracranial complications. ${ }^{15,16}$ Prevention of complications includes effective antibacterial medical therapy to start with to eliminate aerobic and anaerobic organism from the tissues. ${ }^{17}$ Surgical treatment is planned once the organisms are under control. Treatment using non-surgical methods for CSOM includes aural toilet and usage of topical antibiotics. Usage of empirical systemic antibiotics in the treatment of CSOM is practiced by all treating physicians who come across patients with CSOM, be it Paediatricians, ENT surgeons or General Physicians. But the selection of empirical antibiotics, judging their efficacy, resistance to antibiotics and commonly occurring bacteria in CSOM in the community depends upon the knowledge of the physicians. It also depends upon the safety, toxicity and the cost factors of the antibiotics. The culture sensitivity tests used in the present study being in vitro tests are influenced by the microbiology culture yields, multiplicity of organism co-existing in the diseased tissues, variable climatic conditions, patient population and whether antibiotics have or have not been recently used. Several studies have reported different organisms in different proportions. ${ }^{18}$ In the present study, the commonest single organism as well as one among the mixed infections was Staph. aureus MSSA followed by Staph. MRSA (Table 1). A similar study by Gehanno ${ }^{14}$ showed that the next common organism following Staph. MSSA was Pseudomonas. In the present study, Pseudomonas was the third commonest organism found in CSOM and is not in agreement with study in other country. ${ }^{19}$ Amoxicillin alone and in combination with Clavulanic acid were effective against Staph. MRSA organism with sensitivity ranging between 52 and $100 \%$. Ceftazidime and Cephalothin were effective against Pseudomonas in 73.4 and $66.8 \%$ respectively in the present study. Gentamicin was found to have the highest susceptibility rate against all the organism of CSOM ranging from 22 to $86.2 \%$ followed by Chloramphenicol ranging from 18.6 to $71 \%$. Polymyxin was effective by 20 to $68.6 \%$ and its sensitivity against pseudomonas was found to be $58.6 \%$, which was the highest among all other topical antibiotics. Ciprofloxacin was sensitive for CSOM organism ranging from 22 to $72.4 \%$. Ciprofloxacin usage, though previously restricted by few physicians due to its toxic effect on cartilage could be used without apprehensions in view of its efficacy on CSOM organism. 20,21

Another advantage of Ciprofloxacin is that it is not ototoxic and could be used in place of Gentamicin as the former has similar sensitivity range. ${ }^{22}$ In view the high prevalence of S. aureus MSSA and MRSA organisms in CSOM patients in the present study followed by P. aeruginosa and their high susceptibility to Amoxicillin alone and in combination with clavulanic acid and ceftazidime, they could be used in all age groups with safety. However, injudicious use of such drugs can lead to emergence of drug resistant isolates, especially in Staph. aureus and P. aeruginosa ${ }^{23}$ as has previously been experienced; there should therefore be judicious usage of this class of antibiotics in all types of otitis media. The study of microbial pattern and their antibiotic sensitivity determines the prevalent bacterial organisms causing CSOM in the community/local area to start empirical treatment of otitis media and its complications for successful outcome, and thus to prevent the emergence of resistant strains.

\section{CONCLUSION}

The study of microbial pattern and their antibiotic sensitivity determines the prevalent bacterial organisms causing CSOM in the community where empirical antibiotics such as Erythromycin, Ampicillin, Ciprofloxacin, Cephalexin, Clarithromycin and Amoxicillin and Cloxacillin combination are used. The information is useful in treating CSOM and its 
complications for a successful outcome, and thus to prevent the emergence of resistant strains.

\section{REFERENCES}

[1] Jahn AF. Chronic otitis media: diagnosis and treatment. Med Clin North Am 1991;75(6):1277-91.

[2] McPherson B, Holborow CA. A study of deafness in West Africa: the Gambian hearing health project. Int J Pediatr Otorhinolaryngol 1985;10(2):115-35.

[3] Daly KA, Hunter LL, Levine SC, et al. Relationships between otitis media sequelae and age. Laryngoscope 1998;108(9):1306-10.

[4] Tos M. Causes of the disease. Ann Otolaryngol Head Neck Surg 1990;99(4):7.

[5] Tos M. Sequelae of secretory otitis media and the relationship to chronic suppurative otitis media. Ann Otol Rhino Laryngol 1990;99(4):18-9.

[6] Mahoney JL. Mass management of otitis media in Zaire. Laryngoscope 1980;90(7 Pt 1):1200-8.

[7] Kenna MA. Treatment of chronic suppurative otitis media. Otolaryngol Clin North Am 1994;27(3):457-72.

[8] Investing in Health Research and Development. Report of the ad hoc committee on health research funding relating to future intervention options. Geneva: World Health Organization 1996.

[9] Murray CJL, Lopez AD. Deaths by age, sex and cause (thousands) 1990. Global burden of dis-ease. Geneva: World Health Organization 1996:433-68.

[10] Berman S. Otitis media in children. N Eng J Med 1995;332(23):1560-5.

[11] Chotmongkol V, Sangsaard S. Intracranial complications of chronic suppurative otitis media. Southeast Asian J Trop Med Pub Health 1992;23(3):510-3.

[12] Performance standards for antimicrobial susceptibility testing. Tenth informational supplement. National Committee for Clinical Laboratory Standards (NCCLS) 2000;M100S10(M2):14-21.
[13] Acuin J. Chronic suppurative otitis media. BMJ 2002;325(7373):1159-60.

[14] Gehanno P. Multicenter study of the efficacy and safety of oral ciprofloxacin in the treatment of chronic suppurative otitis media in adults. The French study group. Otolaryngol Head Neck Surg 1997;117(1):8390.

[15] Healy GB, Rosbe KW. Otitis media and middle ear effusion in snow. In: Ballenger JJ (edr). Ballenger's otorhinolaryngology head neck surgery. BC Decker inc 2003.

[16] Loy AH, Tan AL, Lu PK. Microbiology of chronic suppurative otitis media in Singapore. Singapore Med J 2002;43(6):296-9.

[17] Brook I. The role of anaerobic bacteria in chronic suppurative otitis media in children: implications for medical therapy. Anaerobe 2008;14(6):297-300.

[18] Sweeney G, Picozzi GL, Browning GG. A quantitative study of aerobic and anaerobic bacteria in chronic suppurative otitis media. J Infect 1982;5(1):47-55.

[19] Agarwal A, Kumar D, Goyal A, et al. Microbiological profile and their antimicrobial sensitivity pattern in patients of otitis media with ear discharge. Indian Jotology 2013;19(1):5-8.

[20] Couzos S, Lea T, Mueller R, et al. Effectiveness of ototopical antibiotics for in aboriginal children, a community based multicentre double blind randomized controlled trial. Med J Aust 2003;179(4):185-90.

[21] Podoshin L, Fradis M, David BJ. Ototoxicity of ear drops in patients suffering from chronic otitis media. J Laryngol Otol 1989;103(1):46-50.

[22] English MG, Northern JL, Fria TJ. Chronic otitis media as a cause of sensorineural hearing loss. Arch Otolaryngol 1973;98(1):18-22.

[23] Gilbert DN, Kohlhepp SJ, Slama KA, et al. Phenotypic resistance of staphylococcus aureus, selected enterobacteriaceae, and pseudomonas aeruginosa after single and multiple in vitro exposures to ciprofloxacin, levofloxacin, and trovafloxacin. Antimicrob Agents Chemother 2001;45(3):883-92. 